\title{
HACIA LA DESGLOBALIZACIÓN Y LA SOBERANÍA ALIMENTARIA
}

\section{GERARDO OTERO}
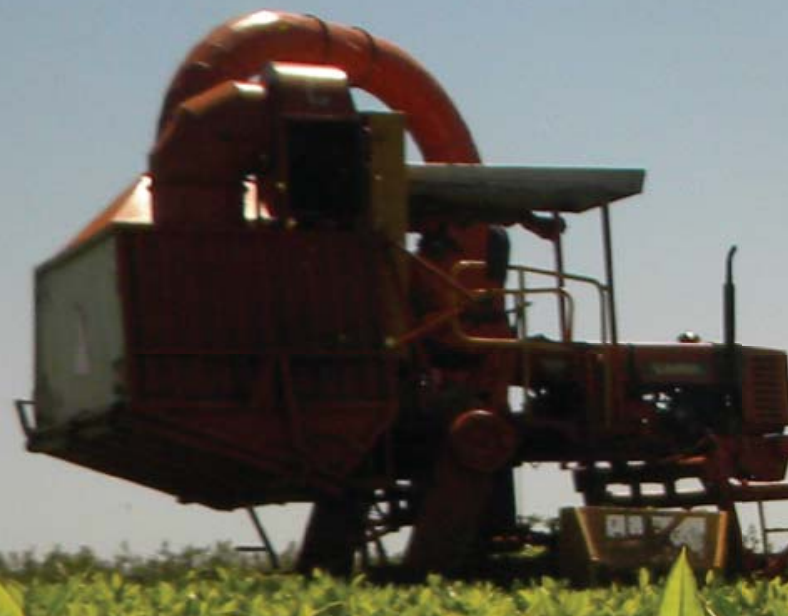

$-\quad 1=-5$

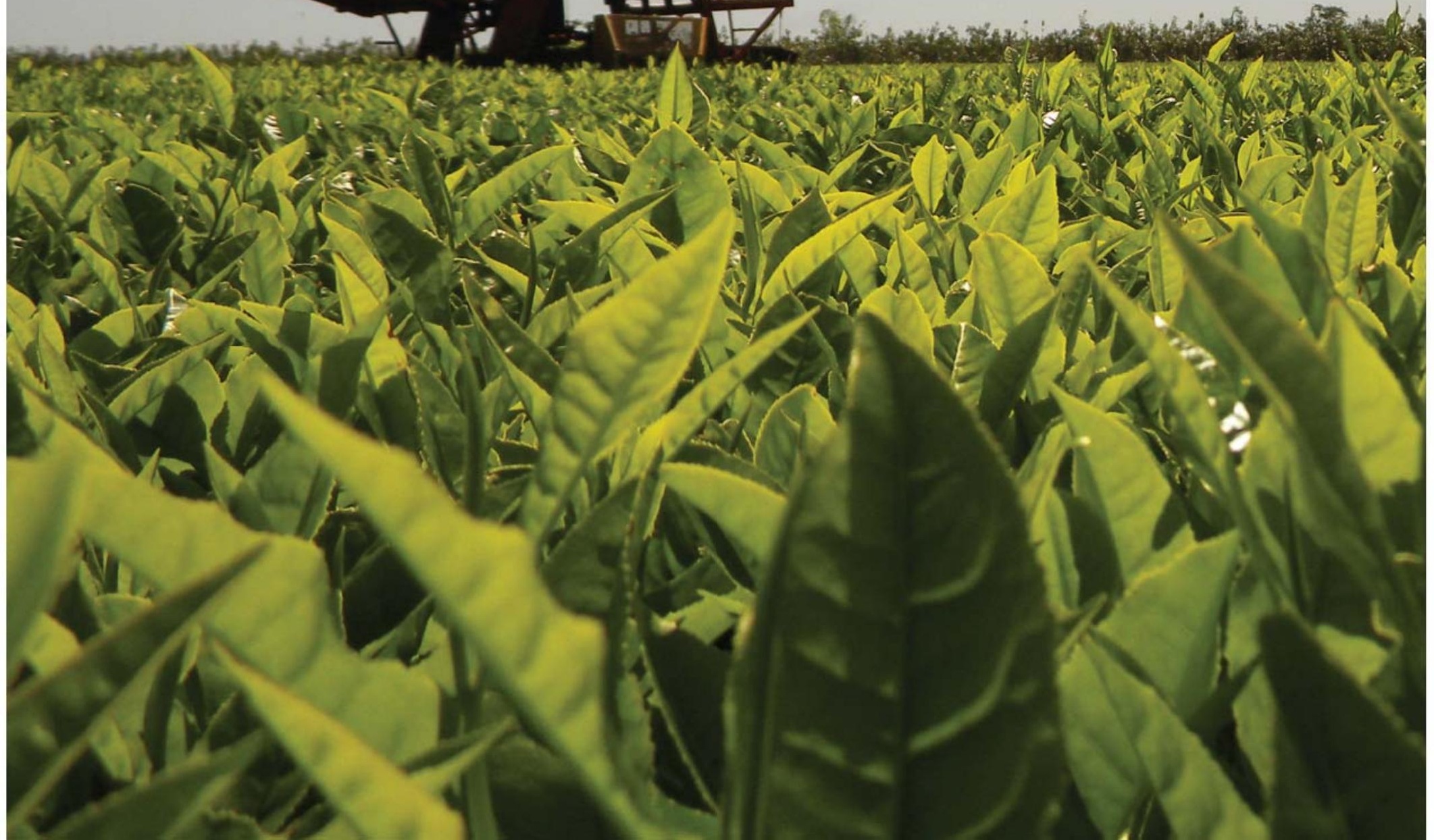


$\mathrm{E}$ 1 mayor logro de Walden Bello en The Food Wars es defender atinadamente el argumento en favor de la desglobalización de la agricultura y la recuperación de la soberanía alimentaria. Ambas metas exigen una relocalización de las economías nacionales en sus mercados internos sin despreciar el comercio internacional. Se trataría, entonces, de deshacerse del carácter neoliberal del capitalismo global y de avanzar hacia una sociedad y una economía radicalmente democráticas en las que sus gentes, en vez de las empresas transnacionales y sus instituciones supraestatales, sean quienes gobiernen el mundo.

Bello nos ofrece un relato maravillosamente accesible de la crisis alimentaria desatada en 2007. No se contenta con desentrañar las principales fuerzas que detonaron la crisis: especulación financiera en los productos agrícolas (commodities) y políticas estadounidenses y europeas encaminadas a promover la producción de agrocombustibles (a los cuales sus defensores llaman eufemísticamente "biocombustibles") como suplemento o sustituto de los hidrocarburos. Bello va más allá de estos factores detonantes de la crisis en busca de sus causas estructurales profundas, de tal forma que se puedan encontrar alternativas de desarrollo adecuadas.

Resulta que los culpables principales de la crisis alimentaria son los paquetes neoliberales de "ajuste estructural”, vigorosamente promovidos desde los años ochenta por las instituciones financieras internacionales (IFI), tales como el Fondo Monetario Internacional, el Banco Mundial, y la Organización Mundial del Comercio (OMC). Mediante la liberalización comercial para las empresas privadas y transnacionales en la agricultura, esas instituciones supraestatales han contribuido enormemente al deterioro de por sí avanzado de los pequeños agricultores y campesinos alrededor del mundo. Así pues, la producción agrícola ha pasado de una lógica centrada en la producción de valores de uso y la reproducción simple de los productores agrícolas, así como la producción de cultivos de calidad, como motivos principales, a la agricultura capitalista moderna, que privilegia los valores de cambio y la ganancia.

Bello plantea sucintamente la disyuntiva futura que plantea la crisis alimentaria: o el paradigma orientado por el mercado o un paradigma centrado en el mercado local (15. Los números en paréntesis se refieren a las páginas en The Food Wars).

El primer capítulo plantea un esbozo del marco teórico para entender el antagonismo entre campesinos y capitalismo; ofrece implícitamente una perspectiva basada en el economista ruso Alexander Chayanov, quien propuso una teoría de la organización campesina de la producción con una lógica distinta de la capitalista. Brinda también una visión de conjunto de la agricultura mundial desde el siglo XIX, basada en la perspectiva

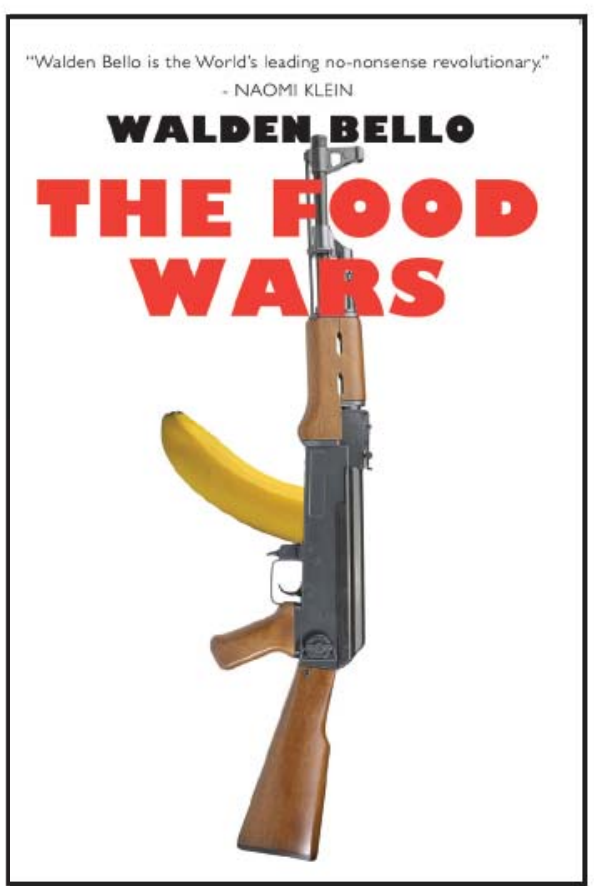

del régimen alimentario desarrollada originalmente por Harriet Friedmann y Philip McMichael (1989). Si bien Reino Unido dominó el primer régimen hasta la Primera Guerra Mundial, Estados Unidos asumió el liderazgo después de la Segunda Guerra Mundial. Un doble rasero de los estadounidenses ha caracterizado el segundo régimen alimentario desde la segunda posguerra: la promoción del libre comercio para todo el mundo, pero imponiendo protecciones para su propio sector agrícola. Esta postura prevalece en las negociaciones agrícolas de la OMC. El avance de las negociaciones de Ronda de Doha de la OMC, iniciada específicamente para concluir la liberalización comercial agrícola, ha sido imposible debido al proteccionismo estadounidense y europeo, combinado con la oposición militante de grupos de oposición como Vía Campesina (Desmarais, 2007), la organización opositora más grande de cualquier clase subordinada a escala transnacional: el campesinado y los pequeños productores agrícolas.

Los siguientes capítulos ofrecen un análisis empírico de la forma en que el neoliberalismo ha impactado al campesinado de varios países. El capítulo dos empieza con una excelente mirada de conjunto de cómo se deterioró el campo mexicano debido a la política estatal. México es, tal vez, el caso paradigmático de la implementación de los "ajustes estructurales" en el sector agrícola. Tal como lo ha descrito un ejecutivo del Banco Mundial citado por Bello, esa política equivalió a un "intervencionismo minucioso sin precedentes" (40). Habiendo sido autosuficiente en 
la producción de alimentos hasta 1989 , a partir de este año, México ha incrementado su dependencia sustancialmente, sobre todo en la importación de granos, cereales y carne desde los Estados Unidos (ver Otero, 2011). Bello resume correctamente tres décadas de neoliberalismo de la siguiente forma: "México se encuentra en un estado de aguda inseguridad alimentaria, crisis económica permanente, inestabilidad política, y una actividad criminal sin control" (53). ¿̨Quién le puede disputar estas conclusiones tras la pérdida violenta de 40,000 vidas desde el inicio de la administración de Felipe Calderón?

El capítulo tres discute el caso de Filipinas, país de donde Bello es originario. Así como para México el alimento básico es el maíz, para Filipinas lo es el arroz. En contraste con el maíz, para el cual existe un gran comercio internacional, en el caso del arroz solamente el $10 \%$ se intercambia en el mercado mundial. La pregunta inicial que plantea Bello sobre Filipinas, como ejemplo de otros países asiáticos que antes gozaron de la autosuficiencia en arroz, es la siguiente: ¿Cómo es que han llegado a ser severamente dependientes de la importación de arroz? (54) La respuesta principal reside nuevamente en los ajustes estructurales de corte neoliberal: Fernando Marcos, dictador filipino hasta 1986, introdujo una reforma agraria moderada y apoyo para los campesinos. Irónicamente, fueron los gobiernos democráticos posteriores los que introdujeron las reformas neoliberales impulsadas por las IFI. El ingreso de Filipinas a la OMC en 1995 tuvo los mismos efectos que el ingreso de México al Tratado de Libre Comercio de América del Norte en 1994, dice Bello (60). Al igual que en México, los precios de dumping del maíz arruinaron a los campesinos, y las reducciones de las tarifas tuvieron efectos perversos para el deterioro de los ingresos fiscales del Estado. Para 2008, unos 20 años después del inicio de la reforma agraria, solamente el $17 \%$ de las tierras que se habían planteado como susceptibles de reforma agraria se habían repartido (65), y la productividad del trabajo se había reducido en relación con la del resto de las economías asiáticas. Los productores filipinos fueron desplazados de cultivo tras cultivo, de manera que su caso es más trágico que el de los campesinos mexicanos.

Pero la destrucción de la agricultura africana fue todavía peor (capítulo cuatro). Si bien África mostró una tasa de crecimiento per capita del ingreso del $3 \%$ entre 1970 y 1973 , para 2007, el Africa SubSahariana había experimentado una reversión de las ganancias que se habían logrado en reducción de la pobreza en 1990-2004. Los ajustes neoliberales aceleraron la espiral descendiente de África (71) y se quedó estancada en una trampa de bajo desarrollo. $\mathrm{Al}$ igual que en México y otros lugares, las mejores tierras se dedicaron a los cultivos de exportación, abandonando los mercados locales a la volatilidad de los precios internacionales.

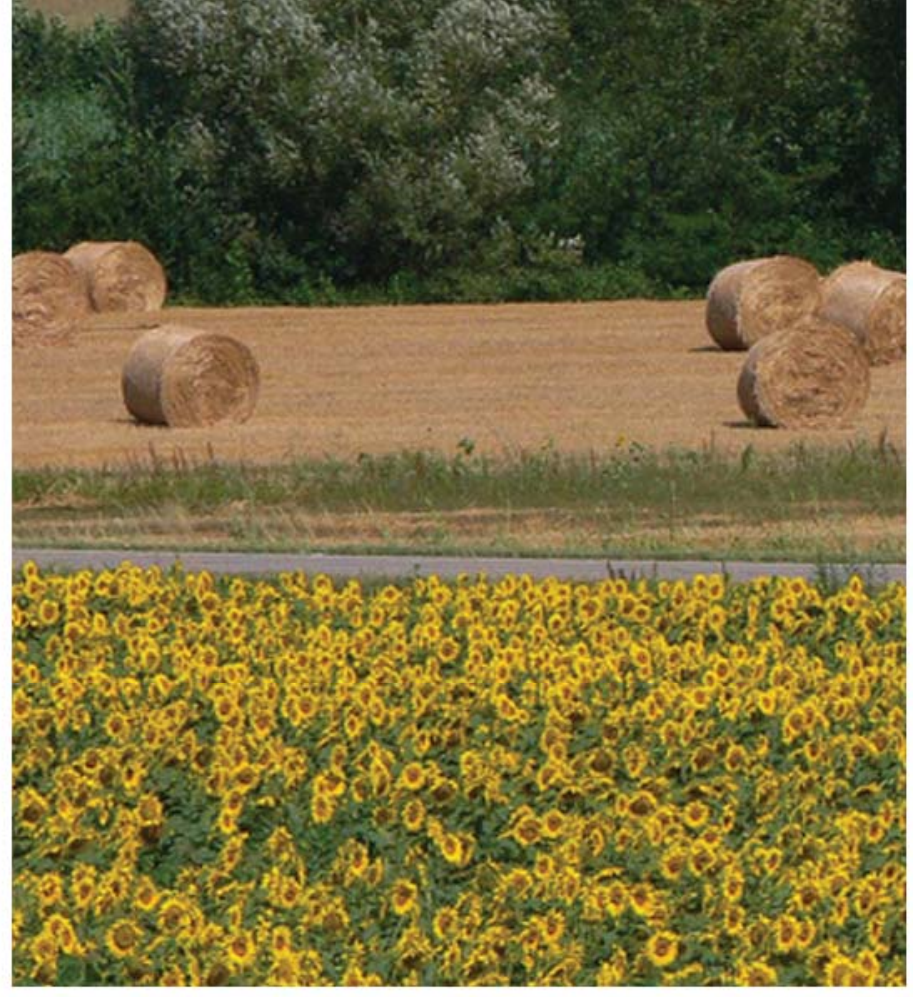

Un notable contraste en cuanto al papel del Banco Mundial (BM) en África es el siguiente: si bien en la mayoría de los países supervisó un "macromanejo" de sus economías, en el sentido de que planteó parámetros estrictos de ajuste estructural; en Africa, implementó de un "micromanejo", en el sentido de que mandó a sus propios agentes a dar seguimiento a las acciones de los funcionarios africanos a cargo de su ejecución. Tuvo que pasar mucho tiempo antes de que el BM reconociera su responsabilidad por el fracaso de los ajustes estructurales; peor aun: diseñó toda una nueva estrategia para establecer reservas laborales, donde habría de montarse programas de reducción de la pobreza para evitar la insurrección. Pero las causas profundas de la crisis quedaron intactas.

El capítulo cinco presenta una síntesis magnífica de la evolución histórica de las relaciones entre el campesinado y el Estado en China. Muestra que, con la excepción de 1978-1984, en que los campesinos vieron un incremento de sus ingresos, el Partido Comunista Chino ha utilizado los excedentes campesinos -y aun partes de sus fondos de reemplazo ya que 30 millones murieron-para financiar y promover la industrialización orientada a las exportaciones. Aun así, China siguió siendo autosuficiente en granos básicos, fue exportadora neta de alimentos por tres décadas y, en 2008, fue el cuarto mayor exportador agrícola mundial. La autosuficiencia alimentaria china, sin embargo, se encuentra bajo grave amenaza debido a cinco cuestiones, nos dice Bello: la creciente carnificación (o creciente dependencia de las carnes) de su dieta; la entrada a la OMC en 2001 la está llevando a incrementar su dependencia alimentaria mediante la descampesinización y el incremento de la importación de granos; los límites ecológicos debidos 
mantener su autonomía respecto de los partidos políticos y su independencia del Estado. Un contraste significativo con el movimiento zapatista en México es que este último se ha enfocado demasiado en sus bases indígenas, en vez de enmarcar su movimiento para que tenga una resonancia y apelación más amplias entre campesinos y trabajadores. Si bien éste fue el intento de "la otra campaña" en 2006, su principal falla fue no haber apoyado tácticamente la campaña de Andrés Manuel López Obrador, en función de mantener su distancia de la política electoral. Ante este vacío a su candidatura, lo que muy probablemente fue un triunfo escamoteado por el fraude podría haber sido una victoria contundente. La izquierda política no perdona a los zapatistas su política de abstención en 2006 y, a partir de ese año, el movimiento ha estado de capa caída (Otero, 2008). Por su parte, el MST ha luchado por lo que Hannah Wittman ha llamado una "ciudadanía ecológica" (2009), o sea, una práctica agraria que convierta a los campesinos en guardianes de la tierra, asegurando el equilibrio ecológico.

En suma, The Food Wars es un libro excelente que recomiendo ampliamente para el uso en clases universitarias de economía, sociología, geografía regional, estudios del desarrollo y de otros campos preocupados por la naturaleza del capitalismo neoliberal. Es accesible también para un público general inquieto por estos temas. No obstante lo anterior, tengo algunos comentarios críticos sobre lapsos conceptuales: cierto esencialismo campesinista en el análisis sobre China, por lo menos una afirmación que no está suficientemente sustentada, y el uso continuo del término "sur global" como concepto, como si tal "región" o entidad existiese en la realidad.

Sobre el primer punto, Bello equipara el plusvalor con la ganancia cuando define el capitalismo como "la organización de la producción para extraer plusvalor o ampliamente, ganancia, de los trabajadores en el proceso de producción" (19). En rigor, la ganancia no es directamente plusvalor, puesto que no todo el capital se invierte en la compra de fuerza de trabajo; también se invierte en capital constante (o muerto). Por tanto, la ganancia debe medirse en relación con la suma de ambos tipos de capital: capital variable (fuerza de trabajo) y capital constante (medios de producción). Un lapso relacionado con el anterior en la misma página es la afirmación de que los campesinos producen principalmente para la subsistencia (lo cual es correcto), "y de manera secundaria para la ganancia monetaria a través de la venta de su plusproducto" (19, GO).

Por definición, sin embargo, el campesinado no produce un plusproducto sino sólo lo suficiente para la reproducción simple. En las ocasiones en que los campesinos producen un plusproducto, pueden ocurrir varias situaciones, sobre todo las siguientes: a) en la mayoría de los casos será apropiado por las clases dominantes o el Estado, b) podrían incrementar o mejorar su nivel de vida mediante un mayor consumo, pero permaneciendo en una situación de reproducción simple, es decir, sin acumulación; o c) el plusproducto se podría convertir en la base material para el aburguesamiento del productor campesino, o sea la contratación sistemática y la explotación de fuerza laboral de trabajadores más allá de los de la unidad doméstica (ver Otero, 2004, capítulo 4).

Estas sutilezas conceptuales son extremadamente importantes para fortalecer el programa de la soberanía alimentaria que propone Walden Bello: si los campesinos, al igual que los capitalistas, persiguiesen "ganancias monetarias”, ¿cuál sería la diferencia entre ellos y los capitalistas para la sociedad? El hecho de que históricamente los campesinos han tenido que convertir una parte de su producción en dinero no se debe a que esa parte representa un plusproducto; más bien, para suplementar su subsistencia, los campesinos requieren otros valores de uso distintos de los que ellos producen directamente. Por ejemplo, los productores de café no pueden vivir de este cultivo solamente. Aun cuando los campesinos tengan una producción diversificada, tendrán necesidad de otros valores de uso, tales como implementos agrícolas, vestido, materiales de construcción, etcétera. Así pues, la lógica de la producción campesina está orientada al aseguramiento de la subsistencia y el reemplazo de su fuerza de trabajo (lo cual incluye la procreación), en vez de la ganancia monetaria per se.

Sin embargo, convendría evitar el esencialismo campesinista. En relación con China, Bello plantea convincentemente que la economía industrial se ha construido sobre las espaldas de los campesinos, y que los ingresos urbanos eran seis veces más altos que los ingresos rurales en el 2000; que los impuestos a los campesinos contribuyeron a un gran crecimiento de la burocracia, etcétera. Se lamenta de cómo China se ha hecho dependiente de la importación de algodón; sin embargo, el algodón no es un cultivo alimentario; por el contrario, podría tratarse de un indicador de que China ha invertido la tradicional relación centroperiferia en la economía mundial: importa productos primarios para generar nuevos empleos en la industria y la exportación de manufacturas. Si bien una gran parte del campesinado se habría convertido en una fuente de fuerza de trabajo excedentaria pauperizada para la industrialización, Bello nunca considera en qué medida los excampesinos se han reencarnado en el proletariado urbano y aun en parte de las crecientes clases medias chinas. 
Bello cita una cifra alarmante acerca de la agricultura moderna: que se lleva muchas más calorías energéticas en insumos de las que produce en calorías alimentarias. El problema son las cifras y sus fuentes. Según la página 36 , la industria agrícola global "emplea tres calorías de energía fósil para producir una caloría de energía alimentaria" (citando un informe del Sierra Club); sin embargo, en la página 140, cita otra fuente según la cual se requieren 10 calorías energéticas para crear sólo una caloría de energía alimentaria entre producción, procesamiento, distribución, y preparación. Dada la seriedad de las cifras y el argumento, habría sido mejor que buscase por lo menos algunas fuentes adicionales para corroborar los datos y evitar las contradicciones entre sus cifras.

Finalmente, parece inconsistente hablar del "sur global" cuando se trata primordialmente de asuntos que tienen que ver con instituciones intergubernamentales como la OMC, por un lado; y de proponer la desglobalización y el programa de soberanía alimentaria que tendrían que ser promovidos por los clases y grupos subalternos, por el otro. La verdad elemental en este asunto es que, además de que algunos agrupamientos efímeros como el "grupo de los 21" en la OMC, el "sur global" existe sólo en la imaginación de ciertos académicos y activistas progresistas. Pero aparte de Vía Campesina, la organización de las sociedades civiles del sur en algo así como una "sociedad civil transnacional" es otro mito (Otero, 2006).

El logro principal de Vía Campesina ha sido contribuir al descarrilamiento de las negociaciones de la OMC, pero los éxitos más tangibles -y positivos- para sus bases se tienen que dar a escala del Estado nación. Como lo planteó Pedro Magaña Guerrero, un campesino mexicano cuya organización es miembro de Vía Campesina, tras exaltar su militancia a escala global: "La consolidación de alternativas depende completamente de lo que pase a escala local, depende del desarrollo de las organizaciones de sus regiones campesinas, en sus países" (citado en Desmarais, 2007: 135). Por tanto, si realmente queremos encaminar el desarrollo y trabajar hacia la desglobalización y relocalización de la producción alimentaria, será mejor poner las prioridades políticas en su lugar: vinculaciones organizativas desde abajo, incluyendo la solidaridad internacional, pero firmemente enraizadas en el Estado nación.

A pesar de las limitaciones mencionadas, The Food Wars es una contribución excepcional para el entendimiento del neoliberalismo y los alimentos, así como una guía perspicaz para la acción transformadora en busca de un futuro sustentable. Lo recomiendo con entusiasmo.
Referencias:

Bartra, Armando y Gerardo Otero (2008), "Rebeldía contra el globalismo neoliberal y el TLCAN en el México rural. Del Estado corporativista a la formación político-cultural del campesinado", Textual, no. 50, pp. 1-34.

Bello, Walden (2002), Deglobalization: Ideas for a New World Economy, London, Zed Books.

Desmarais, A. Annette (2007), La Via Campesina: Globalization and the Power of Peasants, Halifax, Fernwood Press.

Friedmann, Harriet y Philip McMichael (1989), “Agriculture and the State System: The Rise and Decline of National Agricultures, 1870 to the Present", Sociologia Ruralis, no. 29 (2), pp. 93-117.

Otero, Gerardo (1999), Farewell to the Peasantry? Political Class Formation in Rural Mexico, Boulder and Oxford, Westview Press.

Otero, Gerardo (2004), ¿Adiós al campesinado? Democracia y formación política de las clases en el México rural, México, Miguel Ángel Porrúa.

Otero, Gerardo (2006), "Forjando democracia: formación político-cultural y vinculaciones desde abajo”, Íconos. Revista de Ciencias Sociales, Quito, no. 26, pp. 131-146.

Otero, Gerardo (2008), "Contesting neoliberal globalism in Mexico: challenges for the political and the social left", en Paul Bowles, Ray Broomhill, Teresa Gutiérrez-Haces and Stephen McBride (coords.), International Trade and Neoliberal Globalism: Towards Re-Peripheralization in Australia, Canada and Mexico, pp. 163-177, Londres, Routledge.

Otero, Gerardo (2011), "Neoliberal Globalization, NAFTA and Migration: Mexico's Loss of Food and Labor Sovereignty”, Journal of Poverty, no. 15 (4), pp. 384-402.

Wittman, Hannah (2009), "Agrarian Reform and the Environment: Fostering Ecological Citizenship in Mato Grosso, Brazil”, Canadian Journal of Development Studies, no. 29(2-3), pp. 281-298.

Nota: Las publicaciones de Otero se pueden descargar desde su portal: http://www.sfu.ca/ otero. 